\title{
Entanglement creation in circuit QED via Landau-Zener sweeps
}

\author{
Martijn Wubs, ${ }^{1, \oplus}$ Sigmund Kohler, ${ }^{1}$ and Peter Hänggi ${ }^{1}$ \\ ${ }^{1}$ Institut für Physik, Universität Augsburg, D-86135 Augsburg, Germany
}

\begin{abstract}
A qubit may undergo Landau-Zener transitions due to its coupling to one or several quantum harmonic oscillators. We show that for a qubit coupled to one oscillator, Landau-Zener transitions can be used for single-photon generation and for the controllable creation of qubit-oscillator entanglement, with state-of-the-art circuit QED as a promising realization. Moreover, for a qubit coupled to two cavities, we show that Landau-Zener sweeps of the qubit are well suited for the robust creation of entangled cavity states, in particular symmetric Bell states, with the qubit acting as the entanglement mediator. At the heart of our proposals lies the calculation of the exact Landau-Zener transition probability for the qubit, by summing all orders of the corresponding series in time-dependent perturbation theory. This transition probability emerges to be independent of the oscillator frequencies, both inside and outside the regime where a rotating-wave approximation is valid.
\end{abstract}

PACS numbers: 32.80.Qk, 03.67.Lx, 32.80.Bx

\section{INTRODUCTION}

Entanglement is a purely quantum mechanical property of multipartite systems. A system is entangled if its quantum state cannot be described as a direct product of states of its subsystems. Entanglement is measurable in terms of nonclassical correlations of the subsystems. Famous examples are the Einstein-PodolskyRosen correlations between positions and momenta of two particles [1], and the violation of the Bell inequalities by spin systems that are described by Bell states [2]. Fundamental tests of entanglement and nonlocality were performed in quantum optics [3], with the intriguing outcome that measured nonclassical correlations between entangled spatially separated subsystems rule out local realism [4].

With the advent of quantum information theory in recent years, the interest in entanglement has broadened. Many efforts exist to make use of entanglement in information processing or to quantum-communicate with built-in security [5]. With quantum information processing in mind, in this paper we propose to create entanglement in two spatially separated elements (circuit cavities) of a superconducting circuit by letting a third element (the superconducting qubit) undergo a LandauZener sweep. This will be a robust method to create Bell states in two-cavity circuit cavity quantum electrodynamics (QED).

In optical cavity QED, atoms (qubits or $N$-level systems) become entangled with optical cavity modes (quantum harmonic oscillators). The creation of atom-cavity entanglement in cavity QED is possible because the strong coupling regime can be realized, where Rabi oscillations between atomic and optical excitations occur on a faster time scale than spontaneous emission and cavity decay. By adiabatic passage the quantum state of

*Electronic address: martijn.wubs@physik.uni-augsburg.de an atom flying through an optical cavity can be mapped onto the quantum state of the cavity. It was investigated theoretically [6, 7] and shown experimentally [8] that in a similar manner also two modes of the same cavity can be entangled. Alternatively, two spatially separated optical cavities could be entangled by letting an atom fly successively through both cavities 9,10$]$, or by detecting photons leaving the two cavities [11].

If the aim is to build devices for quantum information processing, then scalability to several qubits and oscillators is an important prerequisite. It seems technologically very challenging to scale up optical cavity QED. Recently, a new research field called circuit QED has emerged in which analogues of cavity QED have been realized with superconducting qubits [12, 13] and oscillators. A flux qubit was coupled strongly to a superconducting quantum interference device [14], and a charge qubit to a transmission line resonator [15, 16], and Rabi oscillations have been observed in both experiments. There are analogous proposals to couple superconducting qubits to nanomechanical resonators [17, 18]. Superconducting circuits are promising because of their potential scalability and because many of their parameters are tunable over a broad range.

Scaling up present-day circuit QED can be done by increasing the number of qubits, the number of oscillators, or both. The first option is encountered in most proposals, where the quantum harmonic oscillator is to be used as a bus to manipulate and read out the qubits $[17,[1,19,20]$. The second option is the circuit analogue of two-cavity (or $N$-cavity) QED [9, 10], and the present paper belongs to this category. Also in this group is the recent proposal to couple a superconducting qubit to two transmission-line resonators with orthogonal field polarizations [21]. As to the third option, examples of proposals for experiments with several superconducting qubits coupled to several oscillators can be found in [18, 22]. Probably the first realization of such a complex setup will consist of two qubits independently coupled to their own oscillators, allowing independent qubit state manip- 
ulation and readout.

One method to manipulate the state of an isolated qubit is to use Landau-Zener sweeps [23, 24, 25], as discussed in more detail below. LZ transitions can be used to control qubit gate operations [26, 27] and to read out qubits [28]. Recently, LZ transitions have been observed in various experiments with superconducting qubits [29, 30, 31, 32].

In this paper we concentrate on quantum state manipulation in multi-cavity circuit QED via Landau-Zener sweeps of a qubit. Bit flips in the qubit can take place even in the absence of a direct coupling between the qubit levels, induced instead by the coupling to the oscillators. Oscillators in highly excited coherent states can be described classically and give rise to a non-monotonic LZ transition probability as a function of the coupling strength [33]. Here we focus on the experimentally relevant situation in which the oscillators all start in their ground states. We show how single photons can be created, not only in the presence of only one oscillator as in our prior work [34], and how symmetric Bell states can be created in two circuit oscillators.

The decisive advantage of our proposal is that qubitoscillator interaction strengths are static, in contrast to the situation in standard cavity QED where these interactions require precise dynamical control [7, 9, 20]. Other advantages of LZ transitions are that even adiabatic interactions can be performed rather fast [35], and that LZ transition probabilities are extremely robust under dephasing [36, 37].

In Sec. II we review the standard LZ problem, before deriving in Sec. III exact LZ transition probabilities for a qubit coupled to $N$ harmonic oscillators. This result is used in Sec. IV about single-photon generation and in the central section $\nabla$ about the generation of entangled cavity states via LZ sweeps of the qubit. The realization in circuit QED of our proposals is discussed in Sec. VI. before we conclude.

\section{STANDARD LANDAU-ZENER TRANSITION}

For later use, we shall briefly review the well-known Landau-Zener transition in a qubit. Consider a two-state system with states $|\uparrow\rangle$ and $|\downarrow\rangle$. The standard LandauZener(-Stueckelberg) problem is defined by the Hamiltonian [23, 24, 25]

$$
H(t)=\frac{v t}{2} \boldsymbol{\sigma}_{z}+\Delta \boldsymbol{\sigma}_{x},
$$

where $\boldsymbol{\sigma}_{z} \equiv|\uparrow\rangle\langle\uparrow|-| \downarrow\rangle\langle\downarrow|$ and $\boldsymbol{\sigma}_{x} \equiv|\uparrow\rangle\langle\downarrow|+| \downarrow\rangle\langle\uparrow|$ are Pauli matrices. This Hamiltonian describes how at time $t=0$ the diabatic energies $\pm v t / 2$ of the two states cross with level-crossing speed $v$. During the crossing, the two diabatic states interact with a strength $\Delta$, so that the adiabatic states (or: time-dependent eigenstates) differ from the diabatic states. As is usual, the adiabatic energies are found as the time-dependent eigenvalues of the
Hamiltonian (1). These are $\pm \sqrt{(v t / 2)^{2}+\Delta^{2}}$, showing the archetypical avoided level crossing. The gap between these two adiabatic energies is at least $2 \Delta$, and the minimum occurs at time $t=0$. More intriguing is the dynamics of the state

$$
|\psi(t)\rangle=c_{\uparrow}(t)|\uparrow\rangle+c_{\downarrow}(t)|\downarrow\rangle=\left(\begin{array}{c}
c_{\uparrow}(t) \\
c_{\downarrow}(t)
\end{array}\right),
$$

as described by the Hamiltonian (1). Except around $t=0$, the Hamiltonian (11) is dominated by the diabatic energies. It therefore makes sense to define an interaction picture by the transformation $U_{0}(t)=\exp \left(-\mathrm{i} v t^{2} \boldsymbol{\sigma}_{z} / 2 \hbar\right)$, that is $|\psi(t)\rangle=U_{0}(t)|\tilde{\psi}(t)\rangle$ and $|\tilde{\psi}(t)\rangle=\left[\tilde{c}_{1}(t)|1\rangle+\right.$ $\left.\tilde{c}_{2}(t)|2\rangle\right]$, where the interaction-picture probability amplitudes obey

$$
\frac{\mathrm{d}}{\mathrm{d} t}\left(\begin{array}{c}
\tilde{c}_{\uparrow} \\
\tilde{c}_{\downarrow}
\end{array}\right)=-\frac{\mathrm{i}}{\hbar}\left(\begin{array}{cc}
0 & \Delta \mathrm{e}^{\mathrm{i} v t^{2} / 2 \hbar} \\
\Delta e^{-\mathrm{i} v t^{2} / 2 \hbar} & 0
\end{array}\right)\left(\begin{array}{l}
\tilde{c}_{\uparrow} \\
\tilde{c}_{\downarrow}
\end{array}\right) .
$$

This system of coupled equations of motion (3) is one of the few in driven quantum mechanics that can be solved exactly. The dynamics at all times can be expressed in terms of parabolic cylinder functions 24]. Instead of presenting this dynamics in full glory here, we will give the following useful summary of the dynamics

$$
|\tilde{\psi}(t=\infty)\rangle=\mathrm{S}_{\Delta}|\psi(t=-\infty)\rangle,
$$

in terms of the exact scattering matrix (or S-matrix)

$$
\mathrm{S}_{\Delta}=\left(\begin{array}{cc}
\sqrt{q} & \sqrt{1-q} \mathrm{e}^{-\mathrm{i} \chi} \\
-\sqrt{1-q} \mathrm{e}^{\mathrm{i} \chi} & \sqrt{q}
\end{array}\right) .
$$

Here the quantity $q$ stands for $\exp (-2 \pi \eta)$ and the Stokes phase $\chi=\pi / 4+\arg \Gamma(1-i \eta)+\eta(\ln \eta-1)$, with adiabaticity parameter $\eta=\Delta^{2} /(\hbar v)$ and $\Gamma$ the Euler Gamma function. It follows that the probability $P_{\downarrow}$ that the atom ends up in the initially unoccupied level $|\downarrow\rangle$ is given by

$$
P_{\downarrow} \equiv\left|c_{\downarrow}(t=\infty)\right|^{2}=1-\mathrm{e}^{-2 \pi \Delta^{2} / \hbar v} .
$$

This is the famous Landau-Zener transition probability. It is an exact result for all $\Delta$ and $v$. Instead of using the properties of parabolic cylinder functions to derive this result, which is the standard method, the same transition probability for the standard Landau-Zener problem can be found by exact summation of an infinite series in time-dependent perturbation theory [38]. The latter method is less well known, but it is an important one, since it is this perturbation method that can be used to analyze Landau-Zener transitions in more complex systems as well [37, 39, 40], where an analysis in terms of special functions is not available. An example of a more complex system analyzed by a perturbation series is presented below.

\section{LANDAU-ZENER TRANSITIONS OF A QUBIT COUPLED TO MANY OSCILLATORS}

Next we turn to our main topic, namely LZ sweeps in a qubit that is coupled to $N$ cavity modes. We assume that 
the internal interaction $\Delta$ of the qubit vanishes, unlike in the standard LZ Hamiltonian of Eq. (1), so that all bit flips that occur in the qubit are mediated by the cavity modes. The latter are described as quantum harmonic oscillators. The Hamiltonian becomes

$$
H=\frac{v t}{2} \sigma_{z}+\sigma_{x} \sum_{j=1}^{N} \gamma_{j}\left(b_{j}+b_{j}^{\dagger}\right)+\sum_{j=1}^{N} \hbar \Omega_{j} b_{j}^{\dagger} b_{j} .
$$

By keeping $N$ general here, our calculations are relevant both for setups with one and with two oscillators, on which we focus in later sections. Moreover, in Sec. VI we will show that this Hamiltonian (7) indeed describes the dynamics for LZ sweeps in circuit QED.

We will now calculate the LZ transition probability for a qubit that at time $t=-\infty$ starts in its ground state $|\uparrow\rangle$. We assume that all cavity modes are initially in their ground states $|\mathbf{0}\rangle$ as well, where $|\boldsymbol{n}\rangle$ is a shorthand notation for the Fock state $\left|n_{1}, \ldots, n_{N}\right\rangle$. Since we chose $\Delta=0$ so that the states $|\uparrow\rangle$ and $|\downarrow\rangle$ are eigenstates of the qubit Hamiltonian $\frac{1}{2} v t \sigma_{z}$. Then any transition between the two qubits states can only result from the coupling to the oscillator. Notably, our model (7) is significantly different from the "standard" Landau-Zener problem extended by a bath coupling via $\sigma_{z}[36,41]$.

The central quantity of interest is the probability $P_{\uparrow \rightarrow \downarrow}(t)$ that the qubit has flipped to the state $|\downarrow\rangle$. If the qubit energy is switched slowly (i.e. $v \rightarrow 0$ ), the qubit will follow the adiabatic ground state which at large times is the state $|\downarrow\rangle$; then $P_{\uparrow \rightarrow \downarrow}(\infty)=1$. For large $v$, the qubit will remain in state $|\uparrow\rangle$, so that $P_{\uparrow \rightarrow \uparrow}(\infty)=1$ and $P_{\uparrow \rightarrow \downarrow}(\infty)=0$, corresponding to a nonadiabatic transition. Generalizing the calculation presented in our prior work [34] from one to arbitrarily many oscillators, we derive in the following an exact expression for $P_{\uparrow \rightarrow \uparrow}(\infty)=\sum_{\boldsymbol{n}}|\langle\uparrow, \boldsymbol{n}|U(\infty,-\infty)| \uparrow, \mathbf{0}\rangle|^{2}$ where $U\left(t, t^{\prime}\right)$ denotes the time-evolution operator.

We start by a transformation to an interaction picture with respect to the uncoupled qubit and oscillators,

$$
U_{0}(t)=\exp \left(-\mathrm{i} \sum_{j} \Omega_{j} b_{j}^{\dagger} b_{j} t\right) \exp \left(-\frac{\mathrm{i}}{4 \hbar} v t^{2} \sigma_{z}\right)
$$

which yields the interaction-picture Hamiltonian

$$
\tilde{H}(t)=\sum_{j} \gamma_{j}\left(b_{j}^{\dagger} \mathrm{e}^{\mathrm{i} \Omega_{j} t}+b_{j} \mathrm{e}^{-\mathrm{i} \Omega_{j} t}\right) \exp \left(-\frac{\mathrm{i}}{2 \hbar} v t^{2} \sigma_{z}\right) \sigma_{x} .
$$

Within a perturbation expansion of the probability amplitude $A_{\boldsymbol{n}}=\langle\uparrow, \boldsymbol{n}|U(\infty,-\infty)| \uparrow, \mathbf{0}\rangle$, we obtain the series $A_{\boldsymbol{n}}=\sum_{k=0}^{\infty}(-\mathrm{i} / \hbar)^{2 k} a_{\boldsymbol{n} k}$. Since each $\tilde{H}(t)$ flips the qubit exactly once, only even orders of $\gamma$ appear in $A_{n}$. The $2 k$ th order contribution $a_{\boldsymbol{n} k}$ is characterized by $2 k$ vectors $\boldsymbol{\lambda}_{\ell}, \ell=1, \ldots, 2 k$, where $\boldsymbol{\lambda}$ denotes a vector with exactly one component equal to \pm 1 , while all other components vanish. The $j$ th component $\left(\boldsymbol{\lambda}_{\ell}\right)_{j}= \pm 1$ corresponds to the operators $b_{j}^{\dagger}$ and $b_{j}$, respectively. Then we obtain

$$
\begin{aligned}
a_{\boldsymbol{n} k}= & \sum_{\boldsymbol{\lambda}_{2 k} \cdots \boldsymbol{\lambda}_{1}} C_{n k}\left(\lambda_{2 k}, \ldots, \lambda_{1}\right) \int_{-\infty}^{\infty} \mathrm{d} t_{2 k} \int_{-\infty}^{t_{2 k}} \mathrm{~d} t_{2 k-1} \cdots \int_{-\infty}^{t_{2}} \mathrm{~d} t_{1} \\
& \times \exp \left[\mathrm{i} \sum_{\ell=1}^{2 k} \boldsymbol{\Omega} \cdot \boldsymbol{\lambda}_{\ell} t_{\ell}+\frac{\mathrm{i} v}{2 \hbar} \sum_{\ell=1}^{k}\left(t_{2 \ell}^{2}-t_{2 \ell-1}^{2}\right)\right],
\end{aligned}
$$

where we introduced $\boldsymbol{\Omega}=\left(\Omega_{1}, \ldots, \Omega_{N}\right)$ in order to obtain a more compact vector notation. The appearence of the $\boldsymbol{\lambda}_{\ell}$ in the exponent stems from the sign in the timedependent phase of the creation and annihilation operators. The dots in the coefficient $C_{\boldsymbol{n} k}\left(\lambda_{2 k}, \ldots, \lambda_{1}\right)=$ $\langle\boldsymbol{n}|\cdots| \mathbf{0}\rangle$ denote the combination of $2 k$ operators $\gamma_{j} b_{j}$ and $\gamma_{j} b_{j}^{\dagger}$ that corresponds to the sequence $\boldsymbol{\lambda}_{2 k}, \ldots, \boldsymbol{\lambda}_{1}$. An important simplification of the $\boldsymbol{\lambda}$-summation results from the fact that $C_{\boldsymbol{n} k}=0$ whenever more annihilation than creation operators act on the oscillator ground state $|\mathbf{0}\rangle$. Thus, we need to consider only those $\boldsymbol{\lambda}$-sequences that for all $\ell \leq 2 k$ fulfill the relation

$$
\sum_{\ell^{\prime}=1}^{\ell} \sum_{j}\left(\boldsymbol{\lambda}_{\ell^{\prime}}\right)_{j} \geq 0
$$

For the further evaluation, we substitute in Eq. (10) the times $t_{\ell}$ by the time differences $\tau_{\ell}=t_{\ell+1}-t_{\ell}, \ell=$ $1, \ldots, 2 k-1$, where $t=t_{2 k}$. Thus, we insert $t_{\ell}=t-$ $\sum_{\ell^{\prime}=\ell}^{2 k-1} \tau_{\ell^{\prime}}$ so that the integral in (10) becomes

$$
\begin{array}{r}
\int_{-\infty}^{\infty} \mathrm{d} t \int_{0}^{\infty} \mathrm{d} \tau_{2 k-1} \ldots \mathrm{d} \tau_{1} \exp \left[\mathrm{i} \sum_{\ell=1}^{2 k} \boldsymbol{\Omega} \cdot \boldsymbol{\lambda}_{\ell}\left(t-\sum_{\ell^{\prime}=\ell}^{2 k-1} \tau_{\ell^{\prime}}\right)\right] \\
\quad \times \exp \left[\frac{\mathrm{i} v}{2 \hbar} \sum_{\ell=1}^{k}\left\{2 \tau_{2 \ell-1}\left(t-\sum_{\ell^{\prime}=2 \ell}^{2 k-1} \tau_{\ell^{\prime}}\right)-\tau_{2 \ell-1}^{2}\right\}\right] .
\end{array}
$$

The $t$-integration results in the delta function

$$
2 \pi \delta\left(\frac{v}{\hbar} \sum_{\ell=1}^{k} \tau_{2 \ell-1}+\sum_{\ell=1}^{2 k} \boldsymbol{\Omega} \cdot \boldsymbol{\lambda}_{\ell}\right) .
$$

From the inequality (11) it follows that the second sum in the argument of the delta function is non-negative. Because the integration interval of all $\tau_{\ell}$ is $[0 \ldots \infty)$, any non-zero contribution to the integral (12) results from $\tau_{1}=\tau_{3}=\ldots=\tau_{2 k-1}=0$. Hence, the integral over the time differences $\tau_{2}, \tau_{4}, \ldots, \tau_{2 k-2}$ must yield a distribution proportional to $\delta\left(\tau_{1}\right) \delta\left(\tau_{3}\right) \cdots \delta\left(\tau_{2 k-1}\right)$. Evaluating the integrals over all $\tau_{2 \ell}$ separately, one finds that such a distribution is obtained only if $\sum_{\ell^{\prime}=1}^{2 \ell}\left(\boldsymbol{\lambda}_{\ell^{\prime}}\right)_{j}=0$ for all $\ell=1, \ldots, k-1$. These $k-1$ relations together with the delta function (13) lead to the conditions $\left(\boldsymbol{\lambda}_{2 \ell}+\boldsymbol{\lambda}_{2 \ell-1}\right)_{j}=0$ and hence $\boldsymbol{\lambda}_{2 \ell-1}=-\boldsymbol{\lambda}_{2 \ell}$ for all $\ell=1, \ldots, k$. In combination with Eq. (11) this implies that an integral is non-vanishing only if the non-zero 


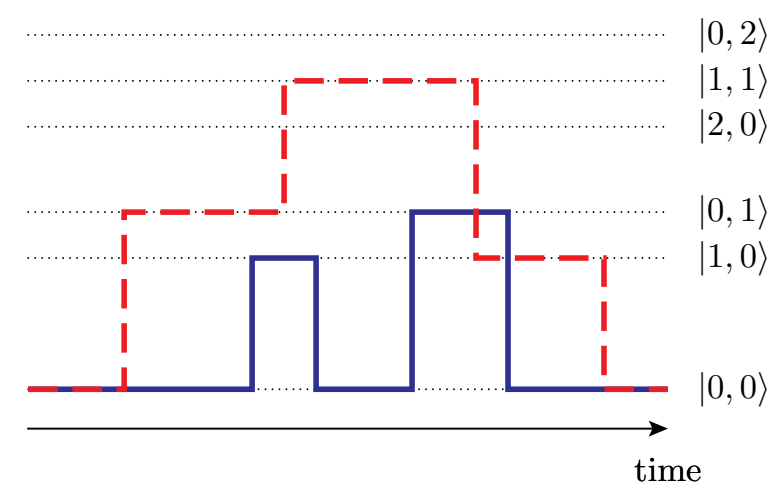

FIG. 1: (Color online) Possible processes during LZ transitions in two-cavity circuit QED. Shown are the lowest-energy levels of the harmonic oscillator states $\left|n_{1}, n_{2}\right\rangle$ for $N=2$. The solid blue line marks a process that contributes to the perturbation series for Landau-Zener transition probability $P_{\uparrow \rightarrow \uparrow}(\infty)$ : the oscillator state jumps (repeatedly) from the ground state to an arbitrary one-photon state and then back to the ground state, in agreement with the no-go-up theorem. In contrast, the process marked by the red dashed line contributes to $P_{\uparrow \rightarrow \uparrow}(t)$ only at finite times.

component of $\boldsymbol{\lambda}_{2 \ell-1}$ is +1 while the same component of $\boldsymbol{\lambda}_{2 \ell}$ equals -1 . In other words, we obtain the selection rule that to the occupation probability at $t=\infty$ only those processes contribute in which the oscillator jumps (repeatedly) from the state $|\mathbf{0}\rangle$ to any state with a single photon (i.e. to $b_{j}^{\dagger}|\mathbf{0}\rangle$ ) and back; see Fig. III. It follows that the oscillators not only start but also end in their ground state $|\mathbf{0}\rangle$ if the final qubit state is $|\uparrow\rangle$. We call this dynamical selection rule the "no-go-up theorem" (see also [39]).

Stating the above result in mathematical terms, of all possible $2 k$-th order processes denoted by sequences of vectors $-\boldsymbol{\lambda}_{2 k-1}, \boldsymbol{\lambda}_{2 k-1}, \ldots,-\boldsymbol{\lambda}_{3}, \boldsymbol{\lambda}_{3},-\boldsymbol{\lambda}_{1}, \boldsymbol{\lambda}_{1}$, the processes that contribute to the perturbation series for $P_{\uparrow \rightarrow \uparrow}(\infty)$ can be characterized by simpler sequences of scalars $j_{k}, \ldots, j_{2}, j_{1}$, where $j_{\ell}$ is the index of the nonvanishing component of both $\boldsymbol{\lambda}_{2 \ell-1}$ and $\boldsymbol{\lambda}_{2 \ell}$. All prefactors $C_{\boldsymbol{n} k}$ of contributing processes have the structure

$$
\begin{aligned}
C_{\boldsymbol{n} k} & =\gamma_{j_{k}}^{2} \ldots \gamma_{j_{3}}^{2} \gamma_{j_{1}}^{2}\left\langle\boldsymbol{n}\left|b_{j_{2 k-1}} b_{j_{2 k-1}}^{\dagger} \ldots b_{j_{1}} b_{j_{1}}^{\dagger}\right| \mathbf{0}\right\rangle \\
& =\gamma_{j_{2 k-1}}^{2} \ldots \gamma_{j_{3}}^{2} \gamma_{j_{1}}^{2} \delta_{\boldsymbol{n}, \mathbf{0}}
\end{aligned}
$$

The remaining multiple integrations are performed as detailed in the appendix of Ref. [38], yielding $a_{\boldsymbol{n} k}=$ $\delta_{\boldsymbol{n}, 0}(\pi \hbar / v)^{k} / k$ ! and is independent of the indices $j_{\ell}$. Therefore the summation over $j_{1}, \ldots, j_{k}$ can be identified as $\left(\sum_{j=1}^{N} \gamma_{j}^{2}\right)^{k}$ so that

$$
A_{\boldsymbol{n}}=\delta_{\boldsymbol{n}, \boldsymbol{0}} \exp [-\pi S / \hbar v)
$$

Consequently, we find the transition probability

$$
P_{\uparrow \rightarrow \downarrow}(\infty)=1-P_{\uparrow \rightarrow \uparrow}(\infty)=1-\mathrm{e}^{-2 \pi S / \hbar v},
$$

in terms of the integrated spectral density

$$
S \equiv \sum_{j=1}^{N} \gamma_{j}^{2}
$$

The final transition probability (16) depends only on $S$ which acts as the effective coupling strength. Notice that quite surprisingly, the transition probability $P_{\uparrow \rightarrow \downarrow}(\infty)$ is independent of the oscillator frequencies $\Omega_{j}$. Nevertheless, the dynamics at intermediate times does depend on them. This was shown to be the case for a qubit coupled to one oscillator in [34], and it will also hold for setups with two oscillators, as presented in Sec. D.

\section{MANIPULATION OF THE SINGLE-OSCILLATOR STATE}

We first consider LZ transitions in the standard cavity QED model of one qubit coupled to one oscillator. Since we start out in the ground state $|\uparrow, 0\rangle$ and the Hamiltonian in Eq. (9) correlates every creation or annihilation of a photon with a qubit flip, the resulting dynamics is restricted to the states $|\uparrow, 2 n\rangle$ and $|\downarrow, 2 n+1\rangle$. As derived above, of the former states only $|\uparrow, 0\rangle$ stays occupied. Thus, the final state exhibits a peculiar type of entanglement between the qubit and the oscillator, and can be written as

$$
\begin{aligned}
|\Psi(\infty)\rangle= & \sqrt{1-P_{\uparrow \rightarrow \downarrow}(\infty)}|\uparrow 0\rangle \\
& +\sqrt{P_{\uparrow \rightarrow \downarrow}(\infty)}\left(c_{1}|\downarrow 1\rangle+c_{3}|\downarrow 3\rangle+\ldots\right),
\end{aligned}
$$

where $\left|c_{1}\right|^{2}+\left|c_{3}\right|^{2}+\ldots=1$. This implies that by measuring the qubit in state $|\downarrow\rangle$, a highly nonclassical oscillator state is produced in which only odd-photon (or: oddphonon) states are occupied. Qubit and oscillator end up fully entangled, in the sense that after tracing out the oscillator states, no coherence between the qubit states $|\uparrow\rangle$ and $|\downarrow\rangle$ is left. The entanglement would have been less perfect if a nonvanishing internal interaction $\propto \boldsymbol{\sigma}_{x}$ between the qubit states $|\uparrow\rangle$ and $|\downarrow\rangle$ had been present [37.

While $P_{\uparrow \rightarrow \downarrow}(\infty)$ is determined by the ratio $\gamma^{2} / \hbar v$, the coefficients $c_{2 n+1}$ depend also on the oscillator frequency. In Fig. 2 we depict how for a small frequency (very small: equal to the coupling strength!) the average photon numbers in the oscillator depend on the state of the qubit. In particular, the average photon number decays rapidly to zero in case the qubit ends "up", in agreement with the no-go-up theorem derived in the previous section, whereas on average more than one photon resides in the oscillator in case the qubit has flipped to $|\downarrow\rangle$. Furthermore, it can be seen that the probability to end up in $|\uparrow\rangle$ indeed tends to the analytically exact final value.

In contrast to the extreme situation depicted in Fig. 2, for the recent experiments in circuit QED [15] and for optical cavity QED one is always in the situation $\gamma \ll \hbar \Omega$, in 


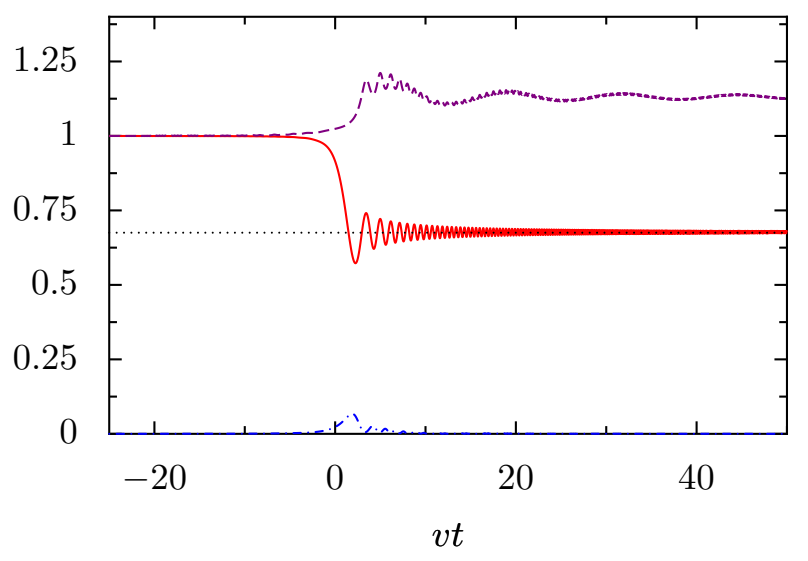

FIG. 2: (Color online) LZ dynamics of a qubit coupled to one oscillator, far outside the RWA regime: $\gamma=\hbar \Omega=0.25 \sqrt{\hbar v}$. The red solid curve is the survival probability $P_{\uparrow \rightarrow \uparrow}(t)$ when starting in the initial state $|\uparrow 0\rangle$. The dotted black line is the exact survival probability $P_{\uparrow \rightarrow \uparrow}(\infty)$ based on Eq. (16). The dashed purple curve depicts the average photon number in the oscillator if the qubit would be measured in state $|\downarrow\rangle$; the dash-dotted blue curve at the bottom shows the analogous average photon number in case the qubit would be measured $|\uparrow\rangle$.

which case $c_{1} \approx 1$ to a very good approximation. Hence one can control via $v$ the final state to be any superposition of $|\uparrow 0\rangle$ and $|\downarrow 1\rangle$. In particular, in the adiabatic limit $v \hbar / \gamma^{2} \ll 1$, the final state becomes $|\downarrow 1\rangle$. This has the important physical implication of the creation of exactly one photon in the cavity, triggered by a Landau-Zener transition. In an experiment, the photon will subsequently leak out of the cavity.

By exploiting these two processes, we propose the following four-step LZ cycle for single-photon generation in circuit QED, as sketched in Fig. 3. The first step is single-photon generation in the cavity via the adiabatic LZ transition $|\uparrow 0\rangle \rightarrow|\downarrow 1\rangle$, brought about by switching the Josephson energy sufficiently slowly. Second, the photon is released from the cavity via the (controlled) cavity decay $|\downarrow 1\rangle \rightarrow|\downarrow 0\rangle$. In the third step, another individual photon is generated via the reverse LZ sweep $|\downarrow 0\rangle \rightarrow|\uparrow 1\rangle$. Fourth and finally, a further photon decay completes the cycle.

This scheme for repeated photon generation via Landau-Zener cycles could be implemented in circuit QED, where the atom-cavity coupling remains at a constant and high value and where qubits are highly tunable so that LZ sweeps can be made from minus to plus an "atomic" frequency, and back. Details about the physical realization in circuit QED of this proposal are deferred to Sec. VI. The strength of this scheme is its simplicity and its robustness against parameter variations, especially variations (and fluctuations [37]) of the oscillator frequency.

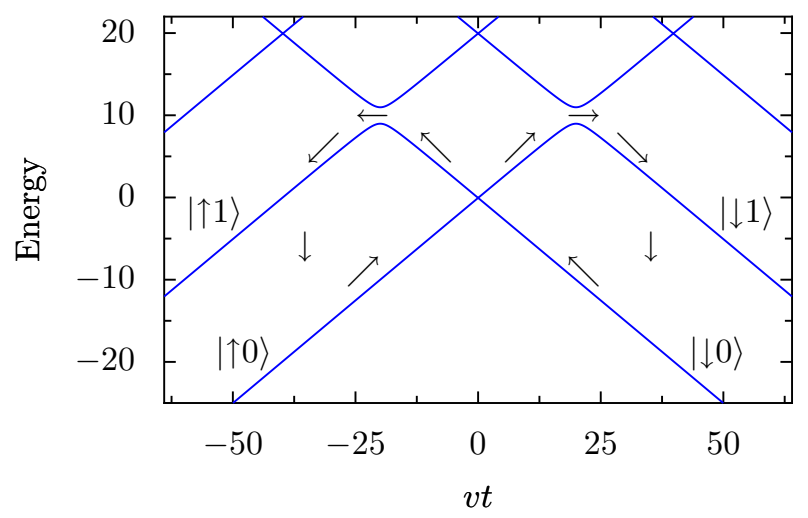

FIG. 3: (Color online) Sketch of adiabatic eigenstates during LZ sweep of a qubit that is coupled to one oscillator. Starting in the ground state $|\uparrow 0\rangle$ and by choosing a slow LZ sweep, a single photon can be created in the oscillator. Due to cavity decay, the 1-photon state will decay to a zero-photon state. Then the reverse LZ sweep creates another single photon that eventually decays to the initial state $|\uparrow 0\rangle$. This is a cycle to create single photons that can be repeated.

\section{ENTANGLING TWO CAVITIES BY A LZ SWEEP OF A QUBIT}

Now consider the situation that the qubit is coupled to two cavities instead of one, with resonance frequencies $\Omega_{1,2}$. We will now show that the two cavity oscillators become entangled by a Landau-Zener sweep of the qubit, and moreover that the specific entangled state in which the oscillators end up can be engineered by varying the Landau-Zener sweep speed $v$ and the frequency detuning $\delta \omega=\left(\Omega_{2}-\Omega_{1}\right)$ of the oscillators.

As before in the case of one oscillator, we will assume $\Delta=0$ for the internal interaction of the qubit, so that all bit flips in the qubit are caused by interactions with the oscillators. For simplicity, we will assume that the two qubit-oscillator strengths are equal, $\gamma_{1}=\gamma_{2}=\gamma$. Without loss of generality, we can take $\Omega_{1} \leq \Omega_{2}$ so that the detuning $\delta \omega$ is nonnegative. The Hamiltonian becomes

$H=\frac{v t}{2} \sigma_{z}+\gamma \sigma_{x}\left(b_{1}+b_{1}^{\dagger}+b_{2}+b_{2}^{\dagger}\right)+\hbar \Omega_{1} b_{1}^{\dagger} b_{1}+\hbar \Omega_{2} b_{2}^{\dagger} b_{2}$.

We will assume that this system starts in the ground state $\left|\uparrow 0_{1} 0_{2}\right\rangle$ before undergoing the Landau-Zener transition. (The subscripts 1,2 that label the two oscillators will be left out below.) The general result (16) then implies that the probability for the qubit to end up in the state $|\downarrow\rangle$ equals

$$
P_{\uparrow \rightarrow \downarrow}(\infty)=1-P_{\uparrow \rightarrow \uparrow}(\infty)=1-\mathrm{e}^{-2 \pi\left(\gamma^{2}+\gamma^{2}\right) / \hbar v} .
$$

At this point it is important to appreciate that this exact result has been derived without making a rotatingwave approximation and by taking the full Hilbert space of the two oscillators into account. The absence of any 


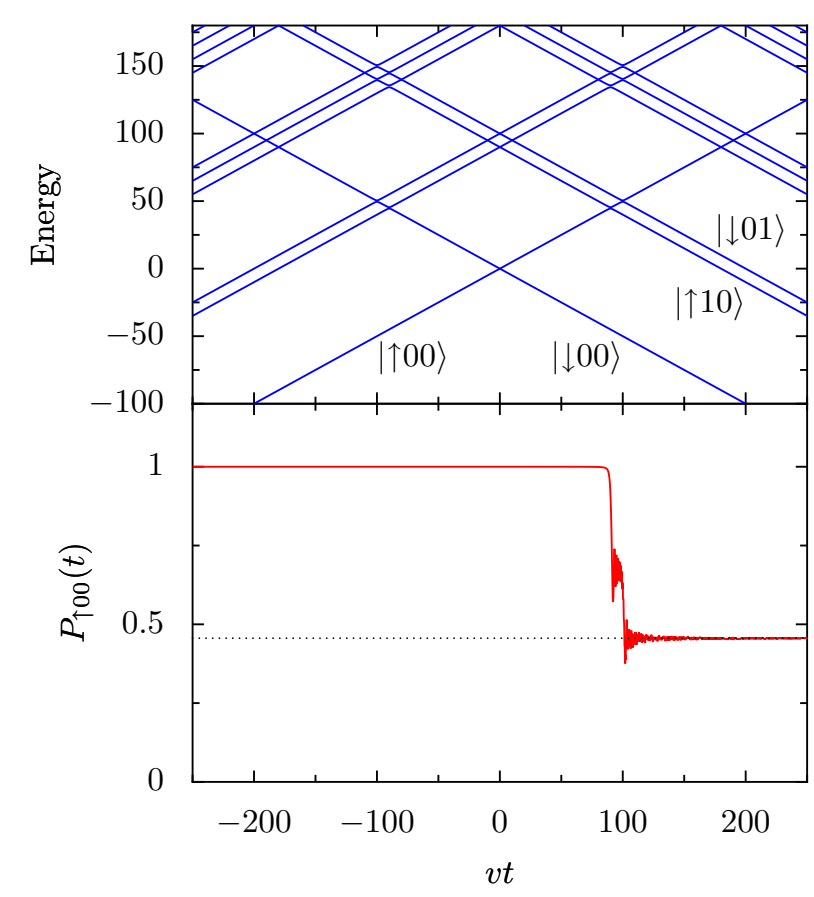

FIG. 4: (Color online) Upper panel: Adiabatic energies during a LZ sweep of a qubit coupled to two oscillators. Parameters: $\gamma=0.25 \sqrt{\hbar v}, \hbar \Omega_{1}=90 \sqrt{\hbar v}$ and $\Omega_{2}=100 \sqrt{\hbar v}$. Viewed on this scale of oscillator energies, the differences between exact and avoided level crossings are invisible. Lower panel: for the same parameters, probability $P_{\uparrow \rightarrow \uparrow}(t)$ that the system stays in the initial state $|\uparrow 00\rangle$ (solid), and corresponding exact survival final survival probability $P_{\uparrow \rightarrow \uparrow}(\infty)$ of Eq. (20) (dotted).

frequency dependence in Eq. (201) is therefore quite surprising.

In the following we are interested in the properties of the final qubit-two-oscillator state $|\psi(\infty)\rangle$ rather than merely the transition probability $P_{\uparrow \rightarrow \downarrow}(\infty)$ of the qubit. In general not much can be said about this final state, but let us now make the realistic assumption $\hbar \Omega_{1,2} \gg \gamma$ : both oscillator energies $\hbar \Omega_{1,2}$ are much larger than the qubitoscillator couplings $\gamma_{1}=\gamma_{2}=\gamma$. Still, the frequency detuning $\delta \omega=\left(\Omega_{2}-\Omega_{1}\right)$ may be larger or smaller than $\gamma / \hbar$. The adiabatic energies in this case are sketched in Fig. 4. As shown in the figure, level crossings that are important for the final state only occur around the times when the qubit energy $v t$ is resonant with one or both of the oscillator energies $\hbar \Omega_{1,2}$. There essentially only three qubit-oscillator states play a role in the dynamics: the initial no-photon state $|\uparrow 00\rangle$ and the two one-photon states $|\downarrow 10\rangle$ and $|\downarrow 01\rangle$. The most general normalized final state can therefore be written as

$$
\begin{aligned}
|\psi(\infty)\rangle & =\sqrt{P_{\uparrow \rightarrow \uparrow}(\infty)}|\uparrow 00\rangle \\
& +\sqrt{P_{\uparrow \rightarrow \downarrow}(\infty)}\left(s_{10}|\downarrow 10\rangle+s_{01}|\downarrow 01\rangle\right),
\end{aligned}
$$

with probabilities $P_{\uparrow \rightarrow \uparrow}(\infty)$ and $P_{\uparrow \rightarrow \downarrow}(\infty)$ given in

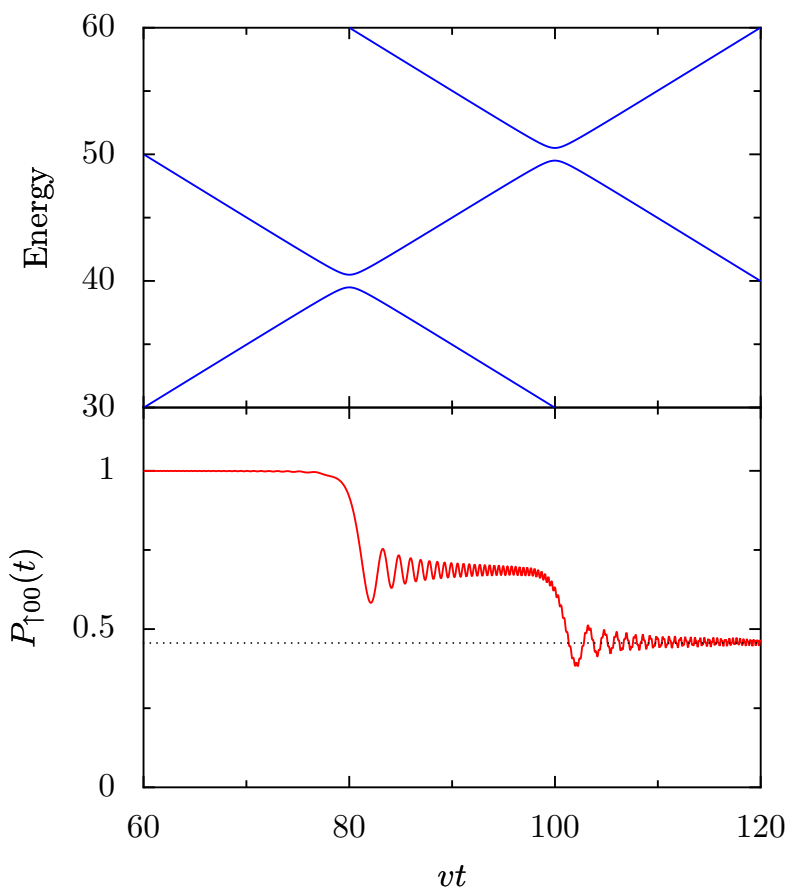

FIG. 5: (Color online) Upper panel: Adiabatic energies during a LZ sweep of a qubit coupled to two oscillators. Parameters: $\gamma=0.25 \sqrt{\hbar v}$ and $\Omega_{2}=100 \sqrt{\hbar v}$, both as in Fig. 四 $\hbar \Omega_{1}=80 \sqrt{\hbar v}$. Lower panel: Probability $P_{\uparrow \rightarrow \uparrow}(t)$ that the system stays in the initial state $|\uparrow 00\rangle$ (solid), and corresponding exact survival final survival probability $P_{\uparrow \rightarrow \uparrow}(\infty)$ of Eq. (20) (dotted).

Eq. (20) and with general complex coefficients $s$ that are only constrained by $\left|s_{10}\right|^{2}+\left|s_{01}\right|^{2}=1$ to ensure state normalization. Below, we will analyze the final state in the limits of large and vanishing detuning of the oscillator frequencies, before addressing the intermediate case $\delta \omega \simeq \gamma / \hbar$.

\section{A. Large detuning $(\delta \omega \gg \gamma / \hbar)$}

If the resonance energies of the cavities differ by much more than the qubit-oscillator coupling, then the dynamics can very well be approximated by two independent standard Landau-Zener transitions, see Figure 5. The first transition occurs when the qubit energy $v t$ is resonant with the energy $\hbar \Omega_{1}$ of the first oscillator. Only the two states $|\uparrow 00\rangle$ and $|\downarrow 10\rangle$ take part in this transition. The other transition occurs around time $t=\hbar \Omega_{2} / v$ and only between the two states $|\uparrow 00\rangle$ and $|\downarrow 01\rangle$.

This situation of two independent transitions is analogous to optical cavity QED with two cavities and one atom flying successively though both of them. The 'time of flight' for the atom between the two cavities here corresponds to the time difference $\delta t=\hbar \delta \omega / v$ between the two resonances.

Just like the standard LZ transition in Sec. III we can 
go to an interaction picture and summarize the transitions by S-matrices. In the basis $\{|\uparrow 00\rangle,|\downarrow 10\rangle,|\downarrow 01\rangle\}$, the $S$-matrix for the first transition becomes

$$
\mathrm{S}_{1}=\left(\begin{array}{ccc}
\sqrt{q^{\prime}} & \sqrt{1-q^{\prime}} \mathrm{e}^{-\mathrm{i} \chi^{\prime}-\mathrm{i} \phi_{1}} & 0 \\
-\sqrt{1-q^{\prime}} \mathrm{e}^{\mathrm{i} \chi^{\prime}+\mathrm{i} \phi_{1}} & \sqrt{q^{\prime}} & 0 \\
0 & 0 & 1
\end{array}\right)
$$

and for the second transition

$$
\mathrm{S}_{2}=\left(\begin{array}{ccc}
\sqrt{q^{\prime}} & 0 & \sqrt{1-q^{\prime}} \mathrm{e}^{-\mathrm{i} \chi^{\prime}-\mathrm{i} \phi_{2}} \\
0 & 1 & 0 \\
-\sqrt{1-q^{\prime}} \mathrm{e}^{\mathrm{i} \chi^{\prime}+\mathrm{i} \phi_{2}} & 0 & \sqrt{q^{\prime}}
\end{array}\right)
$$

Here the quantity $q^{\prime}$ equals $\exp \left(-2 \pi \eta^{\prime}\right)$ with adiabaticity parameter $\eta^{\prime}=\gamma^{2} / \hbar v$. The Stokes phase is now given by $\chi^{\prime}=\pi / 4+\arg \Gamma\left(1-i \eta^{\prime}\right)+\eta^{\prime}\left(\ln \eta^{\prime}-1\right)$, and $\phi_{j}=$ $\hbar \Omega_{j}^{2} / 2 v$. The final state after these two transitions is given by $|\tilde{\psi}(\infty)\rangle=\mathrm{S}_{2} \mathrm{~S}_{1}|\tilde{\psi}(-\infty)\rangle$. Hence, for the initial state $|\tilde{\psi}(-\infty)\rangle=|\uparrow 00\rangle$ we find the final state after two transitions

$$
\begin{aligned}
|\tilde{\psi}(\infty)\rangle & =q^{\prime}|\uparrow 00\rangle \\
& -\sqrt{1-q^{\prime}} e^{\mathrm{i} \theta}\left(|\downarrow 10\rangle+e^{\mathrm{i}\left(\phi_{2}-\phi_{1}\right)} \sqrt{q^{\prime}}|\downarrow 01\rangle\right),
\end{aligned}
$$

with $\theta=\chi^{\prime}+\phi_{1}$. This state is a special case of Eq. (21) in case of two independent LZ transitions. From the final three-level state (24) we find back the exact survival probability $\left|c_{\uparrow}(\infty)\right|^{2}=q^{\prime}=\exp (-2 \pi \eta)$. In the derivation of the exact result (20) we took the full Hilbert space into account rather than a three-level subspace. Moreover, we did not assume whether or not the LZ transitions would occur independently. In other words, the exact probability (20) comes out independent of these two assumptions. Now that the assumptions hold, the exact result will still hold. However, one cannot turn the argument around: finding the survival probability $\left|c_{\uparrow}(\infty)\right|^{2}=q^{\prime}=\exp (-2 \pi \eta)$ does not imply that the rotating-wave approximation was valid after all or that two LZ transitions must have occurred that were independent. Indeed, we will come across a counterexample in Sec. VB.

With the final state (24) determined, one can answer the question whether two successive and independent LZ transitions are a suitable method for entangling the two oscillators. Conditional on measuring the qubit in state $|\downarrow\rangle$, apart from an unimportant overall phase the entangled two-oscillator state becomes

$$
\left|\tilde{\psi}_{\text {osc }}(\infty)\right\rangle=\frac{1}{\sqrt{1+q^{\prime}}}\left(|10\rangle+e^{\mathrm{i}\left(\phi_{2}-\phi_{1}\right)} \sqrt{q^{\prime}}|01\rangle\right) .
$$

In general one would like to be able to create the maximally entangled symmetric Bell state $(|10\rangle+|01\rangle) / \sqrt{2}$. Taking Eq. (25) at face value, the way to obtain equal probabilities for the two one-photon states would be the case $q^{\prime}=1$, but in this case the qubit would never have ended up in $|\downarrow\rangle$ in the first place, as Eq. (24) reveals. So equal probabilities can not be realized. The reason is, for very slow LZ transitions (i.e. for $q^{\prime} \rightarrow 0$ ), all population already follows adiabatically the path $|\uparrow 00\rangle \rightarrow|\downarrow 10\rangle$ in the first transition, so that no population is left to take part in the second LZ transition and hence no population ends up in $|\downarrow 01\rangle$. For faster transitions the situation is less extreme, but a population difference in the final state will remain.

In summary, entangling the two cavities via a LZ sweep of the qubit in the case of large detuning is not ideal, since Bell states cannot be engineered with high probability because of a tradeoff between the probability $P_{\uparrow \rightarrow \downarrow}(\infty)=1-q^{\prime}$ to create at least one photon and the relative probability $q^{\prime} /\left(1+q^{\prime}\right)$ that that one photon ends up in the second cavity. On the other hand, we find hat the state $|\downarrow 10\rangle$ can be created with certainty in the adiabatic limit, which means that single-photon creation in a single oscillator as discussed in Sec. IV can still be realized with LZ sweeps even if another detuned oscillator is present; see also [6].

\section{B. Degenerate oscillator energies $(\delta \omega \ll \gamma / \hbar)$}

Instead of two independent transitions, we will now consider the other extreme case $\delta \omega=0$ so that during the LZ sweep the qubit comes into resonance with both oscillators at the same time. With $\Omega_{1}=\Omega_{2}=\Omega$, the qubit-two-oscillator system has an extra symmetry that we will now exploit in the analysis of the LZ dynamics. Let us first go back and not yet make the rotating-wave approximation. The Hamiltonian (19) now becomes

$$
H=\frac{v t}{2} \sigma_{z}+\gamma \sigma_{x}\left(b_{1}+b_{1}^{\dagger}+b_{2}+b_{2}^{\dagger}\right)+\hbar \Omega\left(b_{1}^{\dagger} b_{1}+b_{2}^{\dagger} b_{2}\right) .
$$

We introduce the new operators

$$
b_{ \pm}=\frac{1}{\sqrt{2}}\left(b_{1} \pm b_{2}\right)
$$

which have standard commutation relations $\left[b_{ \pm}, b_{ \pm}^{\dagger}\right]=1$ and $\left[b_{ \pm}, b_{ \pm}\right]=\left[b_{ \pm}, b_{\mp}^{\dagger}\right]=0$. Both creation operators $b_{ \pm}^{\dagger}$ create one single photon with equal probability in the first or the second oscillator:

$b_{ \pm}^{\dagger}\left|0_{+} 0_{-}\right\rangle=\frac{1}{\sqrt{2}}\left(b_{1}^{\dagger} \pm b_{2}^{\dagger}\right)\left|0_{1} 0_{2}\right\rangle=\frac{1}{\sqrt{2}}\left(\left|1_{1} 0_{2}\right\rangle \pm\left|0_{1} 1_{2}\right\rangle\right)$.

So $b_{+}^{\dagger}$ creates the symmetric and $b_{-}^{\dagger}$ the antisymmetric linear combination. Instead of describing which photon exists in which local oscillator, we can use the fact that a general two-oscillator state can alternatively be written as

$$
\sum_{n_{+}, n_{-}=0}^{\infty} \frac{c_{n_{+} n_{-}}\left(b_{+}^{\dagger}\right)^{n_{+}}\left(b_{-}^{\dagger}\right)^{n_{-}}\left|0_{+} 0_{-}\right\rangle}{\sqrt{\left(n_{+}\right) !\left(n_{-}\right) !}} .
$$


The above rewriting is useful because in terms of the new operators, the Hamiltonian (26) becomes

$$
H=\frac{v t}{2} \sigma_{z}+\sqrt{2} \gamma \sigma_{x}\left(b_{+}+b_{+}^{\dagger}\right)+\hbar \Omega\left(b_{+}^{\dagger} b_{+}+b_{-}^{\dagger} b_{-}\right) .
$$

Note the factor $\sqrt{2}$ in the interaction term. The Hamiltonian (30) shows that the qubit is fully decoupled from the antisymmetric operators. The state of the "antisymmetric photons" will therefore remain unaffected by the LZ sweep. Conversely, whatever the antisymmetric state the two oscillators are in, it will not influence the LZ dynamics. Consequently, for degenerate oscillator frequencies we find back the mathematical problem for a qubit coupled to one oscillator, which we already studied in 34] and in Sec. IV above. The difference lies in the physical meaning of the oscillator, either a local oscillator or a symmetric combination of two local oscillators.

Now assume as before that the initial state is $\left|\uparrow 0_{1} 0_{2}\right\rangle=$ $\left|\uparrow 0_{+} 0_{-}\right\rangle$. Moreover, we take $\hbar \Omega_{1,2}=\hbar \Omega \gg \gamma$ so that the rotating-wave approximation can be made. Then again only the three states $\left|\uparrow 0_{1} 0_{2}\right\rangle,\left|\downarrow 1_{1} 0_{2}\right\rangle$, and $\left|\downarrow 0_{1} 1_{2}\right\rangle$ will play a role in the LZ dynamics. Or, in our new representation, only the two states $\left|\uparrow 0_{+} 0_{-}\right\rangle$and $\left|\downarrow 1_{+} 0_{-}\right\rangle$. The third diabatic state $\left|\downarrow 0_{+} 1_{-}\right\rangle$has a time-dependent energy $(\hbar \Omega-v t / 2)$ and since it is annihilated by the RWA interaction $\sqrt{2} \gamma\left(\sigma_{+} b_{+}+\sigma_{-} b_{+}^{\dagger}\right)$, within the RWA the state $\left|\downarrow 0_{+} 1_{-}\right\rangle$is an adiabatic eigenstate. Indeed, Figure 6] shows that far from the crossing time $v t=$ $\hbar \Omega$ two adiabatic energies overlap with energy values $(\hbar \Omega-v t / 2)$, which is the energy of the diabatic states $\left|\downarrow 1_{1} 0_{2}\right\rangle$ and $\left|\downarrow 0_{1} 1_{2}\right\rangle$, or equivalently, of $\left|\downarrow 1_{+} 0_{-}\right\rangle$and $\left|\downarrow 0_{+} 1_{-}\right\rangle$. At the crossing, one such a diabatic line remains and it corresponds to the state $\left|\downarrow 0_{+} 1_{-}\right\rangle$. The other two states $\left|\uparrow 0_{+} 0_{-}\right\rangle$and $\left|\downarrow 1_{+} 0_{-}\right\rangle$form the avoided crossing in Fig. 6.

The LZ transition probabilities can again be calculated with the help of an $S$-matrix. The $(2 \times 2)$-dimensional S-matrix in the $\left\{\left|\uparrow 0_{+} 0_{-}\right\rangle,\left|\downarrow 1_{+} 0_{-}\right\rangle\right\}$basis gets the form

$$
\mathrm{S}_{+}=\left(\begin{array}{cc}
\sqrt{q^{\prime \prime}} & \sqrt{1-q^{\prime \prime}} \mathrm{e}^{-\mathrm{i} \chi^{\prime \prime}} \\
-\sqrt{1-q^{\prime \prime}} \mathrm{e}^{\mathrm{i} \chi^{\prime \prime}} & \sqrt{q^{\prime \prime}}
\end{array}\right)
$$

where $q^{\prime \prime}=\exp \left(-2 \pi \eta^{\prime \prime}\right)$ and the Stokes phase is given by $\chi^{\prime \prime}=\pi / 4+\arg \Gamma\left(1-\mathrm{i} \eta^{\prime \prime}\right)+\eta^{\prime \prime}\left(\ln \eta^{\prime \prime}-1\right)+\hbar \Omega^{2} / 2 v$, with adiabaticity parameter $\eta^{\prime \prime}=(\sqrt{2} \gamma)^{2} / \hbar v$. It immediately follows that $\left|c_{\uparrow}(\infty)\right|^{2}=q^{\prime \prime}=\exp \left(-4 \pi \gamma^{2} / \hbar v\right)$, again in agreement with the exact result of Eq. (20). Thereby the same generally valid exact result indeed shows up in the two limiting cases that can be treated with simple S-matrices: the case $\left(\hbar \Omega_{1,2} \gg \gamma, \delta \omega \gg \gamma\right)$ in Sec. VA. and here the case $\left(\hbar \Omega_{1}=\hbar \Omega_{2} \gg \gamma\right)$.

The final three-level state in these two limiting cases is different, though. Instead of the state (24), for the qubit coupled to degenerate oscillators starting in $\left|\uparrow 0_{1} 0_{2}\right\rangle$ we now find the final state

$$
|\tilde{\psi}(\infty)\rangle=\sqrt{q^{\prime \prime}}\left|\uparrow 0_{+} 0_{-}\right\rangle-\sqrt{1-q^{\prime \prime}} e^{i \chi^{\prime \prime}}\left|\downarrow 1_{+} 0_{-}\right\rangle
$$$$
=\sqrt{q^{\prime \prime}}\left|\uparrow 0_{1} 0_{2}\right\rangle-\sqrt{1-q^{\prime \prime}} e^{i \chi^{\prime \prime}}\left(\frac{\left|\downarrow 1_{1} 0_{2}\right\rangle+\left|\downarrow 0_{1} 1_{2}\right\rangle}{\sqrt{2}}\right) \text { fll in practice is that the usual fight against decoherence }
$$

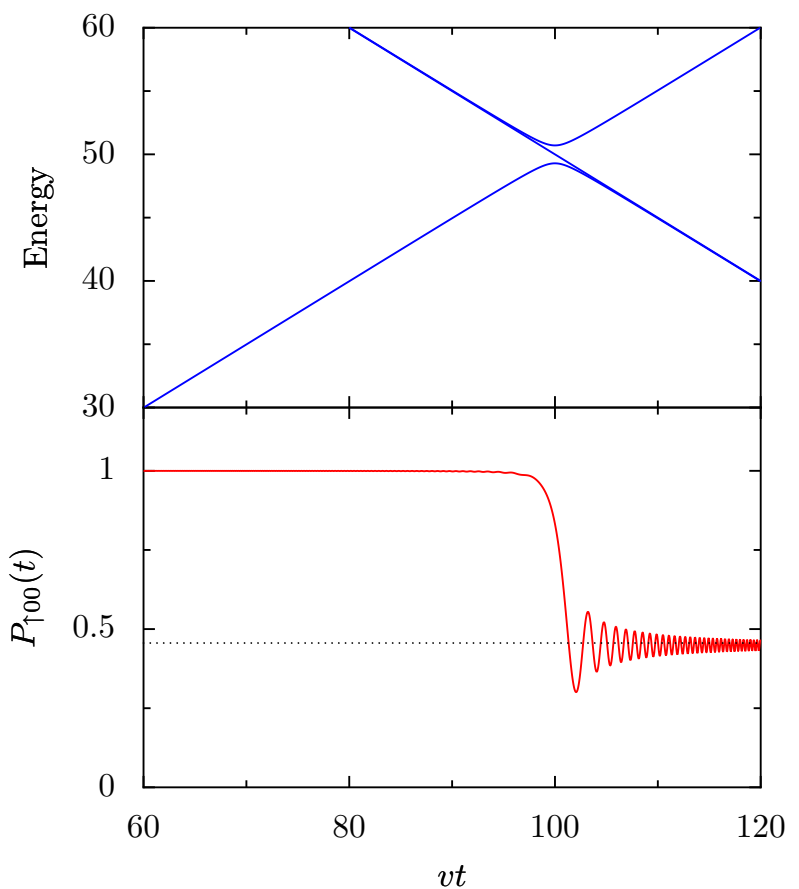

FIG. 6: (Color online) Upper panel: Adiabatic energies during a LZ sweep of a qubit coupled to two oscillators with degenerate energies. Parameters: $\gamma=0.25 \sqrt{\hbar v}$ and $\hbar \Omega_{2}=100 \sqrt{\hbar v}$, as before; this time $\hbar \Omega_{1}=\hbar \Omega_{2}$. Lower panel: Probability $P_{\uparrow \rightarrow \uparrow}(t)$ that the system stays in the initial state $|\uparrow 00\rangle$ (solid), and corresponding exact survival final survival probability $P_{\uparrow \rightarrow \uparrow}(\infty)$ of Eq. (20) (dotted).

Clearly, if the qubit is finally measured and found in state $|\downarrow\rangle$, then the two oscillators end up in the symmetric Bell state

$$
\left|\tilde{\psi}_{\mathrm{osc}}(\infty)\right\rangle=\frac{1}{\sqrt{2}}\left(\left|1_{1} 0_{2}\right\rangle+\left|0_{1} 1_{2}\right\rangle\right) .
$$

Notice that unlike in the final oscillator state (25) after two independent LZ transitions, no relative phase between the two oscillator states $\left|1_{1} 0_{2}\right\rangle$ and $\left|0_{1} 1_{2}\right\rangle$ is built up here. For degenerate oscillators this is as one would expect.

It follows from Eq. (32) that in practice one has two options to produce this Bell state (33): The first option is to employ fast LZ transitions which produce either the sought oscillator Bell state or the oscillator ground state. A measurement of the qubit state is then required, as in a proposal [10] to create Bell states in optical two-cavity QED. If $|\uparrow\rangle$ is measured, then a fast LZ sweep back to the initial ground state is needed and the process can be repeated until the qubit is measured in state $|\downarrow\rangle$, whereby the Bell state is produced. The second option is simpler: one chooses slow LZ transitions and by adiabatic following produces the Bell state (33) with almost certainty.

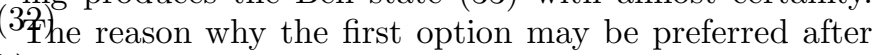
may require faster than adiabatic operations. 


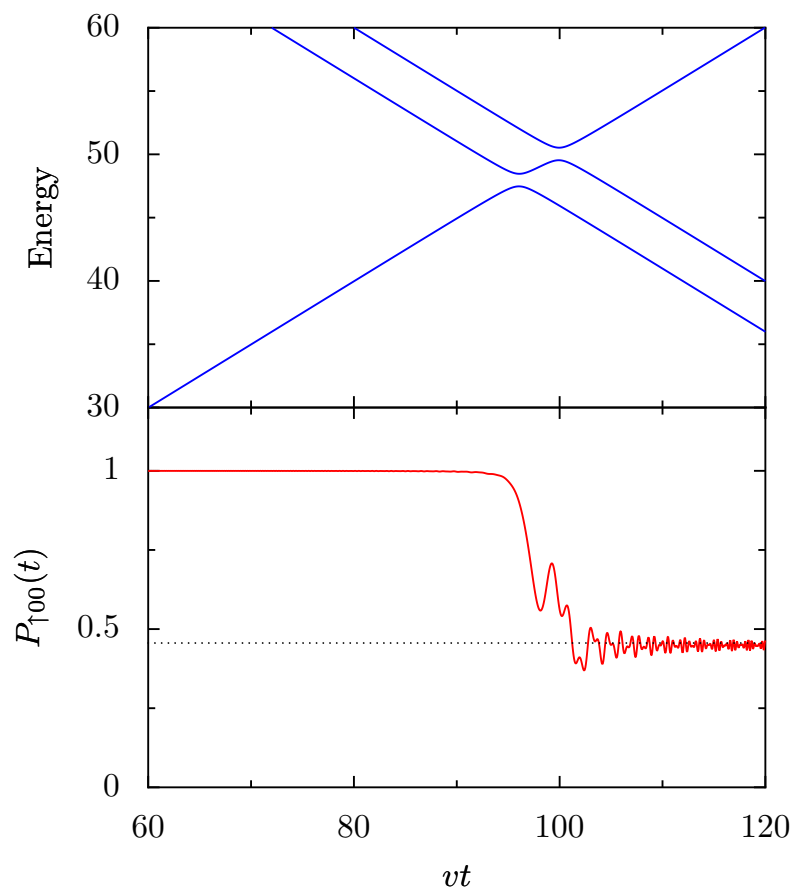

FIG. 7: (Color online) Upper panel: Adiabatic energies during a LZ sweep of a qubit coupled to two oscillators with large energies, and with detunings of the order of the qubit-oscillator coupling $\gamma$. Parameters: $\gamma=0.25 \sqrt{\hbar v}$ and $\hbar \Omega_{2}=100 \sqrt{\hbar v}$, as before; $\hbar \Omega_{1}=96 \sqrt{\hbar v}$. Lower panel: Probability $P_{\uparrow \rightarrow \uparrow}(t)$ that the system stays in the initial state $|\uparrow 00\rangle$ (solid), and corresponding exact survival final survival probability $P_{\uparrow \rightarrow \uparrow}(\infty)$ of Eq. (20) (dotted).

An important difference with the final oscillator state (25) after two independent LZ transitions in $\mathrm{Sec} \mathrm{VA}$ is that in degenerate oscillators always the symmetric Bell state will be produced by measuring the qubit state in $|\downarrow\rangle$, independent of the adiabaticity parameter $\eta^{\prime \prime}$. So if the goal is to produce the symmetric Bell state, then one should try and build systems where a qubit couples with equal strength $\gamma$ to two oscillators with frequency detuning $\delta \omega \ll \gamma$. If on the other hand one would like to be able to vary the final two-oscillator state, then detuned oscillators are to be preferred that give two independent LZ transitions, producing the state (25).

\section{Intermediate detuning $(\delta \omega \simeq \gamma / \hbar)$}

In case of intermediate detuning, the dynamics cannot be described as one or as two independent successive LZ transitions (see Fig. (7). Of course we know from the exact result that the survival $P_{\uparrow \rightarrow \uparrow}(\infty)$ probability is given by Eq. (20), as before. Apart from this probability, we are again interested in the final state. If we assume again that $\hbar \Omega_{1,2} \gg \gamma$ and make the rotating-wave approximation, in the local-oscillator basis we end up with the $(3 \times 3)$
Hamiltonian

$$
H_{\mathrm{RWA}}(t)=\left(\begin{array}{ccc}
v t / 2 & \gamma & \gamma \\
\gamma & \hbar \Omega_{1}-v t / 2 & 0 \\
\gamma & 0 & \hbar \Omega_{2}-v t / 2
\end{array}\right) .
$$

This is a special case of the Demkov-Osherov model [42], in which one level crosses $N$ parallel levels. Interestingly, the transition probabilities (16) and (20) are also exact within the Demkov-Osherov model [42, 43]. Even the scattering matrix $\mathrm{S}$ for the $(N+1)$ levels is known exactly [44, 45]. The interesting result is that the final state is still given by Eq. (24), as if the two level crossings had been independent. Only the phase $\theta$ in Eq. (24) is to be replaced by a more complicated expression [45], but for the two-oscillator state $\left|\tilde{\psi}_{\text {osc }}(\infty)\right\rangle$ this is an irrelevant overall phase. A reason to avoid intermediate detunings in experiments is that convergence of the relative phase to the final value $\left(\phi_{2}-\phi_{1}\right)$ in Eq. (24) is reached more slowly than for large detuning [44], whereas $\left(\phi_{2}-\phi_{1}\right)$ simply vanishes for zero detuning.

In these Secs. $\nabla A \mid \nabla C$ we considered three regimes for the detuning, but we have not yet estimated for which parameters these regimes occur. For example, in practice the two oscillator frequencies will never be exactly degenerate, so the question arises how to define the regime in which they are effectively degenerate. This can be estimated by requiring that the typical time of a single LZ transition $\tau_{\mathrm{LZ}}=2 \gamma / v[33$, 46] is much smaller than the sweeping time $\hbar \delta \omega / v$ from energy $\hbar \Omega_{1}$ to $\hbar \Omega_{2}$. The sweep velocity drops out and we find the requirement $\delta \omega \ll 2 \gamma / \hbar$. Following a similar reasoning, the regime of two independent LZ transitions is characterized by $\delta \omega \gg 2 \gamma / \hbar$.

\section{EXPERIMENTAL REALISATION}

Above we stated that the Hamiltonian (7) can be realized experimentally, and here we briefly outline a realization in circuit QED. Several realizations are promising [14, 15, 16, 17, 18], and here we consider in more detail the setup of the experiments at Yale [15, 16].

We first consider the setup with one qubit and one oscillator, which has already been realized and for which we proposed in Ref. 34] and in Sec. IV] to generate single photons with LZ sweeps. In tunneling representation, a circuit QED setup with one circuit oscillator is described by the Hamiltonian [15, 16]

$H(t)=-\frac{E_{\mathrm{el}}}{2} \sigma_{x}-\frac{E_{\mathrm{J}}(t)}{2} \sigma_{z}+\hbar \Omega b^{\dagger} b+\gamma\left(b^{\dagger}+b\right)\left[\sigma_{x}+1-2 N_{\mathrm{g}}\right]$

for the qubit, the circuit oscillator, and their mutual coupling. The circuit cavity can indeed be modelled as a harmonic oscillator. The electrostatic energy $E_{\mathrm{el}}=$ $4 E_{\mathrm{c}}\left[1-2 N_{\mathrm{g}}\right]$ is determined by the charging energy $E_{\mathrm{c}}$ and the tunable gate charge $N_{\mathrm{g}}$. The tunable flux $\Phi(t)$ penetrating the superconducting loop will be used to 
drive the qubit. The flux controls the Josephson energy $E_{\mathrm{J}}(t)=E_{\mathrm{J}, \max } \cos \left[\pi \Phi(t) / \Phi_{0}\right]$, where $\Phi_{0}$ is the flux quantum. The two-level approximation underlying the Hamiltonian (35) is valid in the "charge regime" $E_{\mathrm{c}} \gg E_{\mathrm{J}}$. In order to minimize decoherence, one typically operates the qubit at the optimal working point $N_{\mathrm{g}}=\frac{1}{2}$, so that $E_{\mathrm{el}}=0$ [47]. Here we also restrict ourselves to this optimal working point. The LZ dynamics can then be realised by switching the flux $\Phi(t)$ in such a way that $E_{\mathrm{J}}(t)=-v t$, with $v>0$. This way, our central Hamiltonian (7) is realized for one qubit coupled to one oscillator $(N=1)$. Moreover, the very low temperatures in circuit QED experiments [15] justify the assumption for our calculations that both the qubit and the oscillator are initially in their ground states, i.e. $|\Psi(-\infty)\rangle=|\uparrow, 0\rangle$, where $\sigma_{z}|\uparrow\rangle=|\uparrow\rangle$.

For an ideal LZ sweep, the Josephson energy should be swept from $E_{\mathrm{J}}=-\infty$ to $E_{\mathrm{J}}=\infty$. In practice, the two-level approximation is valid in a finite energy range. Moreover, in reality $E_{\mathrm{J}}$ is bounded by an $E_{\mathrm{J}, \max }$ which is determined by the critical current. The condition $E_{\mathrm{J}, \max }>\hbar \Omega$ is required so that the qubit comes into resonance with the oscillator sometime during the sweep. The duration of this linear sweep has to be long enough, so that transition probabilities have converged and the finite time interval can be extended to $t=-\infty \ldots \infty$ in calculations describing the dynamics. In circuit QED this situation occurs, since qubit energies can be swept around the oscillator resonance $\hbar \Omega$ over intervals much larger than the interaction $\gamma$.

As another practical condition, inverting the flux through the superconducting loop requires a finite time $2 T_{\min }$, so that $v$ cannot exceed $v_{\max }=E_{\mathrm{J}, \max } / 2 T_{\min }$. For the setup of Refs. [15, 16], the sign of the initial Josephson energy $E_{\mathrm{J}, \max }=2 \pi \hbar \times 10^{10} \mathrm{~Hz}$ can be inverted within $T=1 \mu \mathrm{s}$ so that $v_{\max }=2 \pi \hbar \times 10^{16} \mathrm{~s}^{-2}$.

The cavity frequency $\Omega$ and the qubit-oscillator coupling $\gamma$ are determined by the design of the setup. A typical cavity frequency is $\Omega=2 \pi \times 10^{9} \mathrm{~Hz}$. For the qubit-oscillator coupling strength we assume $\gamma / 2 \pi \hbar=$ $3 \times 10^{6} \mathrm{~Hz}$. Since $\gamma \ll \hbar \Omega$, the generation of more than one photon is negligible. In this limit, by choosing a proper value of $v$, one can obtain any desired superposition of the states $|\uparrow 0\rangle$ and $|\downarrow 1\rangle$, as explored in more detail in 34 .

In Sec. VB we proposed to create Bell states by coupling the qubit with identical couplings $\gamma$ to two degenerate noninteracting circuit oscillators. Such a setup with one qubit and two oscillators currently does not exist, but a detailed proposal how one could fabricate one is given in Ref. [21]. Our wishlist of (almost) identical couplings $\gamma_{1,2}$ and identical resonance frequencies $\Omega_{1,2}$ may not be realized easily. The frequencies are typically are at least $10^{4}$ times larger than the couplings. Ideally one of the two oscillator frequencies is tunable, so that it can be brought into resonance with the other one.

\section{SUMMARY AND CONCLUSIONS}

We have calculated the exact Landau-Zener transition probability of a qubit that is coupled to one or to several quantum harmonic oscillators, given that the system starts in the ground state. The resulting transition probability does not depend on the oscillator frequencies.

The LZ dynamics of a qubit coupled to one oscillator can therefore be manipulated via the sweep speed of the transition. In state-of-the art circuit QED, both adiabatic and nonadiabatic transitions could be realized experimentally by varying the magnetic flux. For different speeds of the transition, the qubit and the oscillator end up in different entangled states, so that LZ transitions can be part of the toolbox to prepare qubit-oscillator entangled states.

Moreover, we have shown how LZ transitions in a qubit can be employed to entangle two oscillators. Especially for oscillators with equal energies, it was found that LZ transitions are a robust way to create in the oscillators the maximally entangled state $(|01\rangle+|10\rangle) / \sqrt{2}$ that is known as the symmetric Bell state. The generation of this particular state requires the qubit to undergo a LZ transition induced by both oscillators at the same time. In circuit QED such a situation may be engineered, for example by coupling two orthogonal transmission-line resonators to the same superconducting qubit [21].

In standard cavity QED, maximally entangled cavity states have been realized experimentally, but only for two degenerate optical modes of the same optical cavity [8]. Our proposal is different in that we consider two spatially separated circuit oscillators, which is somewhat analogous to the optical protocol proposed in Refs. [10, 11]. The difference between the present work and another recent proposal to create Bell states in superconducting circuits [48] is that we consider entanglement creation in non-interacting cavities, whereas in [48] it is shown how Bell states could be created in capacitively interacting qubits.

The decisive advantage of our proposal is that qubitoscillator interaction strengths are static. The archetypical way to entangle two optical cavities is by passing an atom successively through both cavities. This leads to one (non-)adiabatic transition followed by another. Experimentally, there will be a spread in the velocity of the atom when repeating the experiment. The analogue of this situation in circuit QED that we have studied here does not suffer from this disadvantage: a single qubit can be swept through two oscillator resonances either simultaneously or successively, in both cases with a wellcontrolled constant sweep speed.

In principle, our proposal to couple two circuit oscillators to a qubit to create Bell states in them can be generalized to three or more circuit oscillators. For three frequency-degenerate oscillators with equal coupling strength $\gamma$ to the qubit, instead of the symmetric Bell state after the LZ sweep of the qubit, one would 
obtain the so-called W state [49, 50]

$$
|W\rangle=(|001\rangle+|010\rangle+|100\rangle) / \sqrt{3},
$$

while for $N$ oscillators the $N$-qubit W state would be produced [20, 51, 52]. Needless to say that it is technologically very challenging to fabricate such systems, but the creation of these maximally entangled states simply by a single LZ sweep in a qubit would be fascinating.

[1] A. Einstein, B. Podolsky, N. Rosen, Phys. Rev. 47 (1935) 777 .

[2] J. Bell, Physics (Long Island City, N. Y.) 1 (1964) 195.

[3] A. Aspect, P. Grangier, G. Roger, Phys. Rev. Lett. 47 (1981) 460.

[4] M. Redhead, Incompleteness, Nonlocality, and Realism, Clarendon Press, Oxford, 1987.

[5] M. A. Nielsen, I. L. Chuang, Quantum Computing and Quantum Information, Cambridge University Press, Cambridge, 2000.

[6] W. Lange, H. J. Kimble, Phys. Rev. A 61 (2000) 063817.

[7] M. Ikram, F. Saif, Phys. Rev. A 66 (2002) 014304.

[8] A. Rauschenbeutel, P. Bertet, S. Osnaghi, G. Nogues, M. Brune, J. M. Raimond, S. Haroche, Phys. Rev. A 64 (2001) 050301(R).

[9] A. Messina, Eur. Phys. J. D 18 (2002) 379.

[10] D. E. Browne, M. B. Plenio, Phys. Rev. A 67 (2003) 012325 .

[11] D. E. Browne, M. B. Plenio, S. F. Huelga, Phys. Rev. Lett. 91 (2003) 067901.

[12] Y. Nakamura, Y. A. Pashkin, J. S. Tsai, Nature (London) 398 (1999) 786.

[13] C. H. van der Wal, A. J. C. ter Haar, F. K. Wilhelm, R. N. Schouten, C. J. P. M. Harmans, T. P. Orlando, S. Lloyd, J. E. Mooij, Science 290 (2000) 773.

[14] I. Chiorescu, P. Bertet, K. Semba, Y. Nakamura, C. J. P. M. Harmans, J. E. Mooij, Nature (London) 431 (2004) 159.

[15] A. Wallraff, D. I. Schuster, A. Blais, L. Frunzio, R.S. Huang, J. Majer, S. Kumar, S. M. Girvin, R. J. Schoelkopf, Nature (London) 431 (2004) 162.

[16] A. Blais, R.-S. Huang, A. Wallraff, S. M. Girvin, R. J. Schoelkopf, Phys. Rev. A 69 (2004) 062320.

[17] A. N. Cleland, M. R. Geller, Phys. Rev. Lett. 93 (2004) 070501.

[18] M. R. Geller, A. N. Cleland, Phys. Rev. A 71 (2005) 032311.

[19] R. Migliore, K. Yuasa, H. Nakazato, A. Messina, Phys. Rev. B 74 (2006) 104503.

[20] Z. J. Deng, M. Feng, K. L. Gao, Phys. Rev. A 73 (2006) 014302

[21] M. J. Storcz, M. Mariantoni, H. Christ, A. Emmert, A. Marx, W. D. Oliver, R. Gross, F. K. Wilhelm, E. Solano, arXiv:cond-mat/0608526 (2006).

[22] X. L. Zhang, K. L. Gao, M. Feng, Phys. Rev. A 74 (2006) 024303.

[23] L. D. Landau, Phys. Z. Sowjetunion 2 (1932) 46.

[24] C. Zener, Proc. R. Soc. London, Ser. A 137 (1932) 696.

[25] E. C. G. Stueckelberg, Helv. Phys. Acta 5 (1932) 369.

[26] K. Saito, Y. Kayanuma, Phys. Rev. B 70 (2004)

\section{Acknowledgments}

We thank K. Saito and Y. Kayanuma for fruitful discussions. This work has been supported by the DFG through SFB 484, SFB 631, and the cluster of excellence "Nanosystems Initiative Munich".

201304(R).

[27] C. Hicke, L. F. Santos, M. I. Dykman, Phys. Rev. A 73 (2006) 012342.

[28] J. Ankerhold, H. Grabert, Phys. Rev. Lett. 91 (2003) 016803.

[29] A. Izmalkov, M. Grajcar, E. Il'ichev, N. Oukhanski, T. Wagner, H.-G. Meyer, W. Krech, M. H. S. Amin, A. Maassen van den Brink, A. M. Zagoskin, Europhys. Lett. 65 (2004) 844.

[30] G. Ithier, E. Collin, P. Joyez, D. Vion, D. Esteve, J. Ankerhold, H. Grabert, Phys. Rev. Lett. 94 (2005) 057004.

[31] W. D. Oliver, Y. Yu, J. C. Lee, K. K. Berggren, L. S. Levitov, T. P. Orlando, Science 310 (2005) 1653.

[32] M. Sillanpää, T. Lehtinen, A. Paila, Y. Makhlin, P. Hakonen, Phys. Rev. Lett. 96 (2006) 187002.

[33] M. Wubs, K. Saito, S. Kohler, Y. Kayanuma, P. Hänggi, New J. Phys. 7 (2005) 218.

[34] K. Saito, M. Wubs, S. Kohler, P. Hänggi, Y. Kayanuma, Europhys. Lett. 76 (2006) 547.

[35] S. Ashhab, J. R. Johansson, F. Nori, Phys. Rev. A 74 (2006) 052330.

[36] P. Ao, J. Rammer, Phys. Rev. Lett. 62 (1989) 3004.

[37] M. Wubs, K. Saito, S. Kohler, P. Hänggi, Y. Kayanuma, Phys. Rev. Lett. 97 (2006) 200404.

[38] Y. Kayanuma, J. Phys. Soc. Jpn. 53 (1984) 108.

[39] M. V. Volkov, V. N. Ostrovsky, J. Phys. B: At. Mol. Opt. Phys. 38 (2005) 907.

[40] B. E. Dobrescu, N. A. Sinitsyn, J. Phys. B: At. Mol. Opt. Phys. 39 (2006) 1253.

[41] Y. Kayanuma, H. Nakayama, Phys. Rev. B 57 (1998) 13099.

[42] Y. N. Demkov, V. I. Osherov, Zh. Eksp. Teor. Fiz. 53 (1967) 1589, [Sov. Phys. JETP 26 (1968) 916 ].

[43] Y. Kayanuma, S. Fukuchi, J. Phys. B: At. Mol. Phys. 18 (1985) 4089.

[44] S. Brundobler, V. Elser, J. Phys. A 26 (1993) 1211.

[45] J. H. Macek, M. J. Cavagnero, Phys. Rev. A 58 (1998) 348.

[46] K. Mullen, E. Ben-Jacob, Y. Gefen, Z. Schuss, Phys. Rev. Lett. 62 (1989) 2543.

[47] D. Vion, A. Aassime, A. Cottet, P. Joyez, H. Pothier, C. Urbina, D. Esteve, M. H. Devoret, Science 296 (2002) 886.

[48] L. F. Wei, Y.-X. Liu, M. J. Storcz, F. Nori, Phys. Rev. A 73 (2006) 052307.

[49] W. Dür, G. Vidal, J. I. Cirac, Phys. Rev. A 62 (2000) 062314.

[50] P. Walther, K. J. Resch, A. Zeilinger, Phys. Rev. Lett. 94 (2005) 240501. 
[51] H. Häffner, W. Hänsel, C. F. Roos, J. Benhelm, D. ChekAl-Kar, M. Chwalla, T. Körber, U. D. Rapol, M. Riebe, P. O. Schmidt, C. Becher, O. Gühne, W. Dür, R. Blatt, Nature 438 (2005) 643.
[52] R. Doll, M. Wubs, P. Hänggi, S. Kohler, arXiv:cond-mat/0703075 (2007). 